\title{
GAMMA-RAY BURSTS: RESTARTING THE ENGINE
}

\author{
Andrew King, Paul T. O’Brien, Michael R. Goad, Julian Osborne, Emma Olsson, and Kim Page \\ Department of Physics and Astronomy, University of Leicester, Leicester LE1 7RH, UK \\ Received 2005 June 14; accepted 2005 August 3; published 2005 August 17
}

\begin{abstract}
Recent gamma-ray burst observations have revealed late-time, highly energetic events that deviate from the simplest expectations of the standard fireball picture. Instead, they may indicate that the central engine is active or restarted at late times. We suggest that fragmentation and subsequent accretion during the collapse of a rapidly rotating stellar core offers a natural mechanism for this.
\end{abstract}

Subject headings: accretion, accretion disks — black hole physics — gamma rays: bursts

Online material: color figure

\section{INTRODUCTION}

It is now widely believed that gamma-ray bursts (GRBs) lasting longer than about $1 \mathrm{~s}$ are produced by a class of supernovae called "hypernovae" (Paczyński 1998; MacFadyen \& Woosley 1999). Collapse of a massive, rapidly rotating stellar core is assumed to lead to the formation of a black hole, while the remaining core material has enough angular momentum to form a massive accreting neutron torus around it. The infalling torus radiates its gravitational energy as neutrinos or converts it directly to a beam by MHD processes. This evacuates the rotation axis of the core, allowing both an observable burst of gamma rays and the expulsion of a jet of matter at high Lorentz factors. The interaction of this jet with the surroundings produces the X-ray afterglow, which has proved so useful in locating and studying GRBs.

In all cases observed until now, once the initial gamma-ray emission has faded away, the energy output is dominated by the afterglow, which has far lower total energy than the GRB. However, the recent GRB 050502b is quite different (Burrows et al. 2005). An X-ray flare occurred starting some $400 \mathrm{~s}$ after the beginning of the GRB and released at least as much energy as the original burst. There have been several efforts to explain GRB $050502 \mathrm{~b}$ by adding elements to the standard picture of long GRBs, such as late internal shocks or synchrotron selfCompton emission (Burrows et al. 2005; Kobayashi et al. 2005). However, there is considerable difficulty in understanding how GRB 050502b can put so much energy into its surroundings at such late times, and what physical parameter specifies this very different behavior.

Here we consider an idea that offers a way out of these difficulties. In effect, it offers a physical model for the late internal shock suggested by Burrows et al. (2005).

\section{OBSERVATIONS}

GRB 050502b was detected by the Burst Alert Telescope (BAT) on the Swift satellite (Gehrels et al. 2004) at 09:25:40 UT on 2005 May 2 (Falcone et al. 2005). The burst lasted $17.5 \mathrm{~s}$ (T90) with a fluence in the $15-350 \mathrm{keV}$ band of $(8 \pm 1) \times 10^{-7}$ ergs $\mathrm{cm}^{-2}$ (Cummings et al. 2005). The gamma-ray spectrum is well fitted by a single power law with a photon index $\Gamma=1.6 \pm 0.1$. Falcone et al. reported that initially the X-ray count rate seen by Swift was too low to obtain an on-board centroid position with the Swift X-Ray Telescope (XRT) in the $0.3-10 \mathrm{keV}$ band, but subsequent ground-processing revealed an initially fading $\mathrm{X}$-ray source.
This X-ray source brightened dramatically starting some $400 \mathrm{~s}$ after the initial burst, rising to a peak that lasted for several hundred seconds before fading away (Burrows et al. 2005).

Both GRB 050406 (Cummings et al. 2005) and GRB 050607 (Krimm et al. 2005) show behavior similar to that of GRB $050502 \mathrm{~b}$, in that they have a rebrightening of their X-ray emission a few hundred seconds after the initial burst. In both cases, however, the X-ray flares are much weaker relative both to their respective bursts and to the bright flare seen in GRB 050502b. All three of these bursts are relatively low in GRB fluence, particularly GRB 050406, but are not unusually faint. Here we report on an analysis of all three GRBs but concentrate on GRB 050502b. As we do not know the redshifts of these bursts, all physical parameters are quoted in the observer's frame of reference.

The spectrum of the X-ray flare in GRB $050502 \mathrm{~b}$ can be well fitted by a power law with $\Gamma=2.2 \pm 0.03$, but with a time-dependent absorbing column. Similar evidence for spectral variability is seen during the flares for the other GRBs but cannot be well constrained. In GRB 050502b the column is highest at the start of the flare $\left(N_{\mathrm{H}} \approx 1 \times 10^{21} \mathrm{~cm}^{-2}\right.$ above Galactic) and then decreases, strongly suggesting ionization (Burrows et al. 2005). This material was not ionized by the GRB, so presumably it is off the line of sight illuminated by the initial jet. The absorbing column was derived assuming zero redshift, so the intrinsic column will be higher. To derive the X-ray fluence we performed time-dependent spectral fits for GRB 050502b. For the other two bursts, we assumed an intrinsic absorbing column of $N_{\mathrm{H}}=1 \times 10^{21} \mathrm{~cm}^{-2}$ with $\Gamma=2.4$ and 2.3 for GRB 050406 and GRB 050607, respectively.

In Table 1 we quote fluences in the original detector bandpass and those derived by extrapolating the power-law spectra over the $0.3-350 \mathrm{keV}$ bandpass (all bandpasses quoted are in the observer's frame). Extending the energy range to lower energies (e.g., $0.1 \mathrm{keV}$ ) would further enhance the relative strength of the X-ray flare in GRB 050502 b (e.g., it would be $30 \%$ brighter if a low-energy cutoff of $0.1 \mathrm{keV}$ were adopted).

Our spectral analysis of the X-ray flare in GRB 050502b shows that the total fluence of the flare is comparable to or higher than that of the initial GRB (Table 1). This is also illustrated in Figure 1, where we show the flux light curve derived for the XRT bandpass $(0.3-10 \mathrm{keV})$. For the other GRBs the fluence of the X-ray flare is $\leq 10 \%$ of the initial burst. 
TABLE 1

GRB Fluences for the Initial GRB and the X-Ray Flare

\begin{tabular}{cccccc}
\hline \hline GRB & $\begin{array}{c}\text { Burst, } 15-350 \mathrm{keV} \\
\left.\left(10^{-7} \mathrm{ergs} \mathrm{cm}\right)^{-2}\right)\end{array}$ & $\begin{array}{c}\text { Flare, } 0.3-10 \mathrm{keV} \\
\left(10^{-7} \mathrm{erg} \mathrm{cm}^{-2}\right)\end{array}$ & $\begin{array}{c}\text { Burst, } 0.3-350 \mathrm{keV} \\
\left(10^{-7} \mathrm{ergs} \mathrm{cm}^{-2}\right)\end{array}$ & $\begin{array}{c}\text { Flare, } 0.3-350 \mathrm{keV} \\
\left(10^{-7} \mathrm{ergs} \mathrm{cm}^{-2}\right)\end{array}$ & References \\
\hline $050502 \mathrm{~b} \ldots \ldots$ & 8.0 & 8.8 & 11 & 14 & 1,2 \\
$050406 \ldots \ldots$. & 9.0 & 0.9 & 57 & 1.1 & 1,3 \\
$050607 \ldots \ldots$. & 8.9 & 1.1 & 17 & 1.5 & 1,4 \\
\hline
\end{tabular}

ReferenCES. - (1) This paper; (2) Cummings et al. 2005; (3) Krimm et al. 2005; (4) Retter et al. 2005.

\section{ENERGETICS}

We now consider how the central engine in a GRB might restart. Our line of argument is straightforward. The original GRB in 050502b signaled the formation of a compact object (neutron star or black hole) and the accretion of a stellar mass onto it on a very short timescale, presumably from a neutron torus. The simplest explanation for the comparable energy of the X-ray flare is that a second, similar mass accreted after a delay $t_{d}$ of up to $400 \mathrm{~s}$ (allowing for possible light-travel effects). Clearly this large mass cannot have already been in the first torus or it would have been accreted along with it. Thus a second stellar-mass torus must form and accrete after $t_{d}$.

In two-dimensional models of fallback the extra mass is already in a torus, by construction, and the delay between the GRB and X-ray flare in GRB 050502b would have to reflect some viscous angular momentum transport timescale before the torus became dynamically unstable and rapidly accreted. In contrast, if we do not assume high symmetry, an obvious possibility is that this mass is a second compact "star" (i.e., a self-gravitating neutron lump), formed because the collapsing core had enough angular momentum to fragment. Gravitational radiation then drags the lump in toward the first compact object, where tides smash it up into a torus that can be accreted, releasing an energy comparable to the original GRB. This effectively amounts to restarting, or reactivating, the central engine at late times.

Restarting the GRB central engine also accounts for the extraordinarily rapid rate of increase and decrease in the X-ray flare, which goes as greater than $t^{7}$ if we use the time $(t)$ of the initial burst as the origin, $t=0$. Such rates are hard to accommodate in the standard fireball model of GRB afterglows

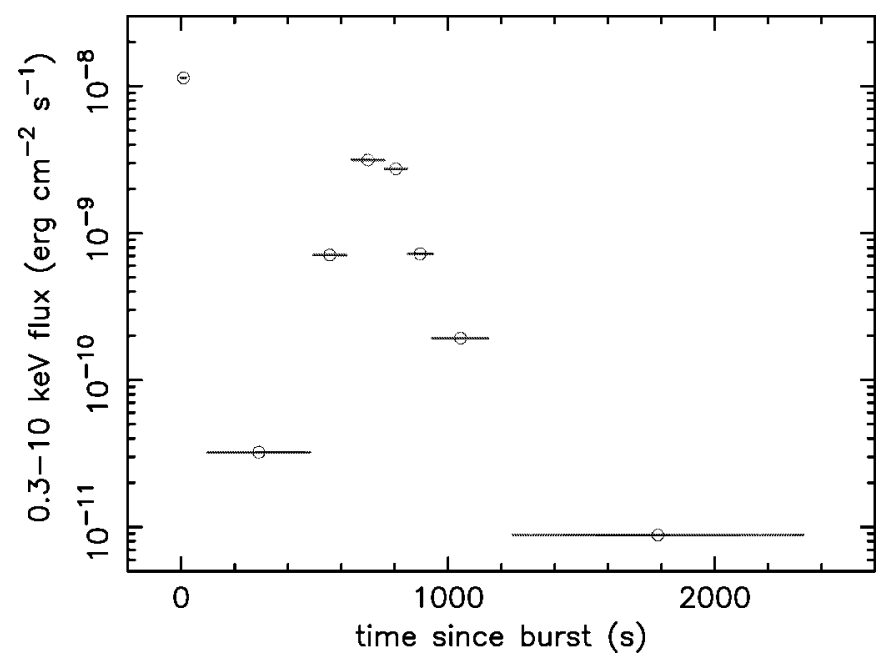

FIG. 1.-Unabsorbed $0.3-10 \mathrm{keV}$ flux light curve for the early phase of GRB 050502b. The horizontal bars represent the time intervals over which the fluxes were calculated. The first point is the initial GRB extrapolated to the 0.3-10 keV band. [See the electronic edition of the Journal for a color version of this figure.] and instead strongly support a reactivated central engine. The BAT on Swift did not detect gamma rays from the X-ray flare in GRB 050502b, possibly because the flare spectrum was soft and the energy was spread over many seconds.

\section{CORE COLLAPSE AND FRAGMENTATION}

The two-stage collapse described above is almost exactly that envisaged by Davies et al. (2002) to explain why hypernovae are rare among supernovae, and any possible delays between supernovae and GRBs. Davies et al. (2002) argued by comparison with theoretical studies of star formation (see Bonnell \& Pringle 1995) that the collapse of a rapidly rotating stellar core is likely to lead to fragmentation and the initial formation of more than one compact object of nuclear density. They considered a case where the collapses producing these objects make a supernova rather than an initial GRB, i.e., where none of them is surrounded by an accreting torus. Depending on the way fragmentation occurs, cases where the initial collapse produces a GRB are clearly possible.

The compact objects subsequently coalesce under the effect of gravitational radiation. Provided that at least one of them is a neutron star rather than a black hole, Davies et al. show that there must be a stage where a neutron torus accretes onto a central object, thus releasing an energy comparable to the initial burst. Equation (7) of that paper shows that gravitational radiation emission drags an orbiting fragment in, to the point where it disrupts and forms a neutron torus, on a timescale

$$
\tau_{\mathrm{GR}}=0.18 \times \frac{\left(a_{0} / 1000 \mathrm{~km}\right)^{4}}{m_{1} m_{2}\left(m_{1}+m_{2}\right)} \mathrm{hr} .
$$

Here $a_{0}$ is the initial circular orbit radius and the central black hole and orbiting fragment have masses $m_{1} M_{\odot}$ and $m_{2} M_{\odot}$, respectively. This gives a gravitational radiation delay timescale $\tau_{\mathrm{GR}}=0.18 \mathrm{hr}=640 \mathrm{~s}$ if the merging masses are $\sim 1 M_{\odot}$ and their initial separation is $a_{0}=1000 \mathrm{~km}$, which in turn requires a specific angular momentum $j=10^{17} \mathrm{~cm}^{2} \mathrm{~s}^{-1}$, exactly what hypernova models require. This will be true for any required gravitational radiation delay (which is also affected by the redshift) since $j \propto a^{1 / 2} \propto \tau_{\mathrm{GR}}^{1 / 8}$. One can arrange the gravitational radiation delay to be shorter than the observed delay if the $\mathrm{X}$ ray event corresponds to a second faster outflow overtaking the initial one, although a substantial overtaking delay is rather contrived.

The initial orbit of the fragment may be somewhat misaligned from the spin axis of the central black hole. Tidal dissipation in the orbiting fragment and viscous dissipation in the neutron torus cause rapid alignment through the Lense-Thirring effect (see King et al. 2005). The second burst (X-ray flare) thus has a jet axis close to the first one. This may explain why the flare is spectrally softer and lasts longer than the original GRB. In the internal shock picture, the peak spectral energy scales as $E_{p} \propto \Gamma^{-2} t_{v}^{-1}$ (e.g., Table 2 of Zhang \& Mészáros 
2002), where $\Gamma$ is the bulk Lorentz factor and $t_{v}$ is the variability timescale of the outflow. The cleaner environment for the second jet may reduce its baryon loading and thus increase $\Gamma$. It is also possible that tidal effects make the second accretion event smoother than the first (increasing $t_{v}$ ), although a full hydrodynamic calculation is needed to check this.

\section{SMALLER X-RAY FLARES}

Several other GRBs have been observed to have significant X-ray flares superposed on their fading afterglows (see Table 1 and Piro et al. 2005), although none are nearly as bright or quite as late as seen in GRB 050502b. GRB 050502b itself has another, smaller X-ray event $\approx 10^{5} \mathrm{~s}$ after the burst (Burrows et al. 2005) with a fluence similar to that of the event in GRB 050607 (some 10\% of the initial GRB). These smaller flares may be evidence of a similar process involving the accretion of smaller neutron clumps by the central object: for a sufficiently complex fragmentation process there may even be several events. A lower limit to the relative luminosity of such smaller events to the main GRB arises from the result (Davies et al. 2002) that the minimum mass of a neutron-rich clump is $\sim 0.2 M_{\odot}$ (smaller masses make nuclei in their centers). The energies of a merger event to the original GRB must be in the ratio $r=\eta_{2} M_{2} / \eta_{1} M_{1}$, where $M_{1}$ and $M_{2}$ are the masses of the first compact object and the subsequent merging object, and $\eta_{1}$ and $\eta_{2}$ are the efficiencies of the collapse and merger events. For comparable efficiencies a merger must have $r \gtrsim 0.2 M_{\odot} / M_{1} \gtrsim 0.02$ (for $M_{1} \lesssim 10 M_{\odot}$ ). The observed X-ray/GRB ratio depends on the spectral index of the emitted flux.

\section{DISCUSSION}

The observed fluence of the late X-ray flare in GRB 050502b is comparable to that of the main GRB and is spectrally softer. Smaller but possibly related flares have also been seen in other bursts. Whether the unusually bright X-ray flare event in GRB $050502 \mathrm{~b}$ is unique or simply an extreme example of a pattern of behavior common to GRBs is unclear. For example, the time of the late X-ray flare in GRB 050502b is within the known range of GRB durations (e.g., Paciesas et al. 1999), which extends to at least $1000 \mathrm{~s}$. Many bursts, but not all, show spectral evolution such that they become softer later (e.g., Norris et al. 1986). It has also been shown that if a GRB engine is quiescent for a long time, the subsequent emission outburst could be unusually large (Ramirez-Ruiz \& Merloni 2001). The fragmentation picture that we have suggested here may be able to explain these phenomena. Continuing accretion at a slower rate from a fragment may be the origin of the long, faint X-ray afterglows seen in many Swift GRBs, which probably require the central engine to remain on for a long time (see Zhang \& Mészáros 2001). We will investigate this possibility in a future paper.

We have suggested that X-ray flares may result from the fragmentation of the collapsing stellar core and the subsequent merger of a significant fragment with the most massive compact object formed in the collapse. This is a departure from the current hypernova picture, in which only one object is assumed to form directly in the collapse. However, the rapid rotation apparently required to make a hypernova is known to lead to fragmentation in other situations such as star formation. A simple test of our idea will come from combining GRB observations with gravitational-wave detections by the Laser Interferometer Gravitational-Wave Observatory. Long GRBs should be significant sources of gravitational radiation, with a chirp signal characteristic of a merging binary system. If this proves successful it would also give a clean determination of the masses involved, providing major insight into the process of core collapse in rapidly rotating stars.

We thank Evert Rol for useful discussions and the referee for a very helpful report. A. K. acknowledges a Royal SocietyWolfson Research Merit Award. Emma Olsson is supported by a European Union Research and Training Network grant. The Swift project and theoretical astrophysics research at the University of Leicester are supported by PPARC.

\section{REFERENCES}

Bonnell, I. A., \& Pringle, J. E. 1995, MNRAS, 273, L12

Burrows, D. N., et al. 2005, Science, submitted

Cummings, J. R., et al. 2005, GCN Circ. 3339, http://gen.gsfc.nasa.gov/gen/ $\operatorname{gcn} 3 / 3339 . \mathrm{gcn} 3$

Davies, M. B., King, A. R., Rosswog, S., \& Wynn, G. A. 2002, ApJ, 579, L63

Falcone, A., et al. 2005, GCN Circ. 3330, http://gcn.gsfc.nasa.gov/gcn/gcn3/ 3330.gen3

Gehrels, N., et al. 2004, ApJ, 611, 1005

King, A. R., Lubow, S. L., Ogilvie, G. G., \& Pringle, J. E. 2005, MNRAS, in press (astro-ph/0507098)

Kobayashi, S., Zhang, B., Mészáros, P., \& Burrows, D. N. 2005, ApJ, submitted
Krimm, H., et al. 2005, GCN Circ. 3183, http://gcn.gsfc.nasa.gov/gcn/gcn3/ 3183.gen 3

MacFadyen, A. L., \& Woosley, S. E. 1999, ApJ, 524, 262

Norris, J. P., et al. 1986, ApJ, 301, 213

Paciesas, W. S., et al. 1999, ApJS, 122, 465

Paczyński, B. 1998, ApJ, 494, L45

Piro, L., et al. 2005, ApJ, 623, 314

Ramirez-Ruiz, E., \& Merloni, A. 2001, MNRAS, 320, L25

Retter, A., et al. 2005, GCN Circ. 3525, http://gcn.gsfc.nasa.gov/gcn/gcn3/ 3525.gen 3

Zhang, B., \& Mészáros, P. 2001, ApJ, 552, L35

. 2002, ApJ, 581, 1236 